\title{
The rise of new technologies for aortic valve stenosis: A comparison of sutureless and transcatheter aortic valve implantation
}

\author{
Augusto D'Onofrio, MD, ${ }^{\mathrm{a}}$ Stefano Salizzoni, MD, ${ }^{\mathrm{b}}$ Antonino S. Rubino, MD, ${ }^{\mathrm{c}}$ Laura Besola, MD, ${ }^{\mathrm{a}}$ \\ Claudia Filippini, MD, ${ }^{\mathrm{b}}$ Ottavio Alfieri, MD, ${ }^{\mathrm{d}}$ Antonio Colombo, MD, ${ }^{\mathrm{d}}$ Marco Agrifoglio, MD, \\ Theodor Fischlein, MD, ${ }^{\mathrm{f}}$ Filippo Rapetto, MD, ${ }^{\mathrm{g}}$ Giuseppe Tarantini, MD, ${ }^{\mathrm{a}}$ Magnus Dalèn, MD, \\ Davide Gabbieri, MD, ${ }^{i}$ Bart Meuris, MD, ${ }^{\mathrm{j}}$ Carlo Savini, MD, ${ }^{\mathrm{k}}$ Giuseppe Gatti, MD, \\ Marco Luigi Aiello, MD, ${ }^{\mathrm{m}}$ Fausto Biancari, MD, ${ }^{\mathrm{n}}$ Ugolino Livi, $\mathrm{MD},{ }^{\circ}$ Pier Luigi Stefàno, $\mathrm{MD},{ }^{\mathrm{p}}$ \\ Mauro Cassese, MD, ${ }^{\mathrm{q}}$ Bruno Borrello, MD, ${ }^{\mathrm{r}}$ Mauro Rinaldi, MD, ${ }^{\mathrm{c}}$ Carmelo Mignosa, MD, ${ }^{\mathrm{b}}$ and \\ Gino Gerosa, MD, ${ }^{a}$ on behalf of the Italian Transcatheter Balloon-Expandable Registry and the Sutureless \\ Aortic Valve Implantation Research Groups
}

\begin{abstract}
Objective: Transcatheter aortic valve implantation (TAVI) and sutureless aortic valve replacement (SU-AVR) are suitable alternatives to conventional surgery. The aim of this study is to compare early outcomes of patients undergoing TAVI and SU-AVR.

Methods: Data were analyzed on patients who underwent TAVI and patients who underwent SU-AVR. Two matched cohorts (TAVI vs SU-AVR) were created using propensity scores; all analyses were repeated for transapical TAVI and transfemoral TAVI, separately. Outcomes were defined according to Valve Academic Research Consortium-2 criteria.
\end{abstract}

Results: A total of 2177 patients were included in the analysis: $1885(86.6 \%)$ treated with TAVI; $292(13.4 \%)$ treated with SU-AVR. Mortality in unmatched TAVI and SU-AVR patients was $7.1 \%$ and $2.1 \%$, respectively, at 30 days, and $12.9 \%$ and $4.6 \%$, respectively, at 1 year. No differences were found in 30-day mortality in the 214 matched patient pairs $(3.7 \%$ vs $2.3 \% ; P=.4)$, but patients treated with TAVI showed a lower incidence of device success $(85.9 \%$ vs $98.6 \% ; P<.001)$ and pacemaker implantation $(2.8 \%$ vs $9.4 \% ; P=.005)$, and a higher incidence of any paravalvular leakage (PVL).

Conclusions: SU-AVR is associated with better device success and a lower incidence of PVL, compared with TAVI. Nevertheless, patients treated with SU-AVR were more likely to receive a permanent pacemaker. SU-AVR and TAVI provide good results in patients who have severe symptomatic aortic valve stenosis. Given the multiple therapeutic options available, patients may receive the treatment that is most appropriate for their clinical and anatomical characteristics. ( $\mathrm{J}$ Thorac Cardiovasc Surg 2016;152:99-109)

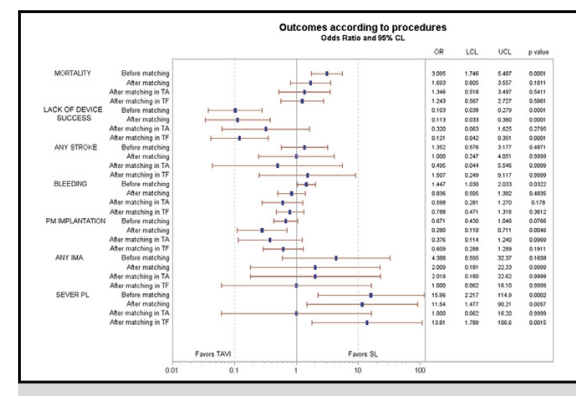

A forest plot showing outcomes according to procedure (TAVI and sutureless) and TAVI access approach.

\section{Central Message}

In a propensity-matched analysis, SU-AVR, compared with TAVI, had better device success and a lower incidence of PVL but similar 30 -day and 1-year mortality.

\section{Perspective}

The search for the ideal valve substitute for "intermediate-risk" patients (the so-called "grey zone") is ongoing. This propensity-matched analysis shows that SU-AVR and TAVI are both good solutions. TAVI provides better gradients and a shorter length of stay, but SU-AVR has less PVL. These results may provide help in choosing the best therapeutic option for each patient.

See Editorial Commentary page 110

\footnotetext{
From the ${ }^{\mathrm{a} D i v i s i o n}$ of Cardiac Surgery, University of Padova, Padova; ${ }^{\mathrm{b}}$ Department of Cardiac Surgery, University of Turin-Città della Salute e della Sceinza-Molinette, Turin; ${ }^{\mathrm{c}}$ Department of Cardiac Surgery, AOU-Policlinico Vittorio Emanuele, University of Catania, Catania; ${ }^{\mathrm{d}}$ Department of Cardiac Surgery, San Raffaele Hospital, Milan; ${ }^{\mathrm{e}}$ Department of Cardiac Surgery, University of Milan-Monzino Hospital, Milan, Italy; ${ }^{\mathrm{f}}$ Department of Cardiac Surgery, Nürnberg Clinic, Nürnberg, Germany; ${ }^{\mathrm{g}}$ Department of Cardiac Surgery, IRCCS San Martino-National Institute for Cancer Research, Genova, Italy; ${ }^{\mathrm{h}}$ Department of Cardiac Surgery, Karolinska Institute, Karolinska University Hospital, Stockholm, Sweden; ${ }^{i}$ Department of Cardiac Surgery, Hesperia Hospital, Modena, Italy; ${ }_{\mathrm{j}}^{\mathrm{j}}$ Department of Cardiac Surgery, University Hospital Gasthuisberg, Leuven, Belgium; ' ${ }^{\mathrm{D}}$ epartment of Cardiac Surgery, Azienda Ospedaliera Universitaria S. Orsola, Bologna; ${ }^{\mathrm{D}}$ Department of Cardiac Surgery, Ospedali Riuniti, Trieste; ${ }^{\mathrm{m}}$ Department of Cardiac Surgery, IRCCS Policlinico S. Matteo, Pavia, Italy;
}

${ }^{\mathrm{n}}$ Department of Cardiac Surgery, Oulu University Hospital, Oulu, Finland; ${ }^{\circ}$ Department of Cardiac Surgery, AOU S. Maria Misericordia, Udine; ${ }^{\mathrm{P}}$ Department of Cardiac Surgery, AOU Careggi, Firenze; ${ }^{\mathrm{q}}$ Department of Cardiac Surgery, S. Maria Hospital, Bari; and ${ }^{\mathrm{r} O s p e d a l e ~ M a g g i o r e, ~ P a r m a, ~ I t a l y . ~}$

Read at the 95th Annual Meeting of The American Association for Thoracic Surgery, Seattle, Washington, April 25-29, 2015.

Received for publication May 5, 2015; revisions received Oct 16, 2015; accepted for publication Nov 17, 2015; available ahead of print Feb 19, 2016.

Address for reprints: Augusto D'Onofrio, MD, Division of Cardiac Surgery, University of Padova, Centro Gallucci, Via Giustiniani 2, 35128 Padova, Italy (E-mail adonofrio@hotmail.it).

$0022-5223 / \$ 36.00$

Copyright (c) 2016 by The American Association for Thoracic Surgery

http://dx.doi.org/10.1016/j.jtcvs.2015.11.041 


\section{Abbreviations and Acronyms \\ AVR = aortic valve replacement \\ EuroSCORE $=$ European system for cardiac operative risk evaluation \\ ITER $=$ Italian Transcatheter Expandable-Balloon Registry \\ PARTNER trial $=$ Placement of Aortic \\ Transcatheter Valve trial \\ PVL = paravalvular leakage \\ SU-AVR = sutureless aortic valve \\ TAVI $=$ transcatheter aortic valve implantation \\ TA-TAVI $=$ transapical TAVI \\ TF-TAVI $=$ transfemoral TAVI}

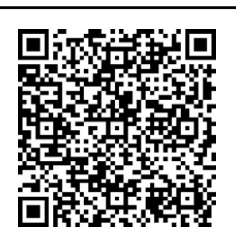

Although aortic valve replacement (AVR) is the treatment of choice for patients suffering from severe symptomatic aortic valve stenosis, new therapeutic options provide interesting results. Transcatheter aortic valve implantation (TAVI) has shown very good clinical and hemodynamic outcomes for up to 5 years in high-risk or inoperable patients. $^{1-3}$ Based on these outcomes, TAVI is now recommended by the guidelines in patients who are deemed inoperable or who have a prohibitive risk (class I, level of evidence B) and is considered a reasonable alternative in high-risk patients (class IIa, level of evidence B) ${ }^{4}$ Furthermore, investigators are now comparing AVR to TAVI in intermediate-risk patients, as the trend toward an extension to TAVI indications is ongoing. ${ }^{5,6}$ Sutureless aortic valve replacement (SU-AVR) allows the replacement of the diseased aortic valve, after resection of the aortic cusps and complete decalcification of the annulus, without the need for annular sutures for prosthesis anchoring. This approach enables a faster positioning of the valve prosthesis, thereby reducing aortic crossclamp, as well as cardiopulmonary bypass, time $\mathrm{T}^{7-9}$; in addition, it is an easier approach with minimally invasive techniques. In fact, many authors have reported their experiences using SU-AVR with ministernotomy or right mini-thoracotomy. ${ }^{10,11}$ Thus, TAVI and SU-AVR both should be considered good therapeutic alternatives to AVR in patients who have aortic valve stenosis. The aim of the current multicenter study was to compare early outcomes of TAVI and SU-AVR in patients who have severe aortic valve stenosis.

\section{METHODS}

Patient-informed consent for treatment, data collection, and analysis for scientific purposes was collected in all cases. The local ethics committees approved the data collection relating to patients undergoing TAVI and SU-AVR.

\section{Transcatheter Aortic Valve Implantation}

Data from the ITER (Italian Transcatheter Balloon-Expandable TAVI Registry) registry were used for this study. The ITER is a real-world all-comers spontaneous registry that includes patients who underwent TAVI with the balloon-expandable Sapien and Sapien XT bioprostheses (Edwards Lifesciences, Irvine, Calif) at 33 centers in Italy, between 2007 and 2012 (Appendix 1). Indications for TAVI were the presence of any of the following: (1) severe symptomatic aortic valve stenosis (defined as an aortic valve area $<0.8 \mathrm{~cm}^{2}$ and a mean transaortic gradient $>40 \mathrm{~mm}$ $\mathrm{Hg}$ ) in high-surgical risk patients (ie, those with a logistic European System for Cardiac Operative Risk Evaluation [EuroSCORE] of $\geq 20 \%$; (2) predicted risk of mortality $\geq 10 \%$, as defined by the Society of Thoracic Surgeons; and (3) unsuitability for AVR because of porcelain aorta or serious comorbidities, including chronic kidney failure, chronic obstructive pulmonary disease, previous total chest irradiation, hostile chest, and severe liver disease. A multidisciplinary TAVI team that included at least a cardiac surgeon, an interventional cardiologist, and one anesthesiologist, evaluated all cases.

\section{Sutureless Aortic Valve Replacement}

Data from a European Multicenter study group that includes patients who underwent isolated SU-AVR with a Perceval bioprosthesis (Sorin Biomedica, Saluggia, Italy) at any 1 of 6 European centers, between 2010 and 2014, were analyzed (Appendix 2). The implantation of this sutureless bioprosthesis was considered feasible if the aortic annulus size was 19 to $27 \mathrm{~mm}$, and if the ratio between the diameter of the sinotubular junction and the diameter of the aortic annulus did not exceed 1.3. The ascending aorta was incised transversally $1.5 \mathrm{~cm}$ above the sinotubular junction. The aortic valve was removed, and the annulus was completely decalcified. The appropriate valve size was selected using the manufacturer sizing tool. Three $4 / 0$ polypropylene guiding sutures were passed at the nadir of each aortic sinus. Simultaneously, an appropriate-size prosthesis was collapsed on a separate table and firmed into the manufacturer holder. The 3 guiding sutures were passed through the 3 eyelets at the inflow ring of the prosthesis, which was consequently positioned into the aortic anulus. The aortic valve was released and the holder removed. The prosthesis was dilated with a balloon, at 4 atmospheres for 30 seconds, while the field was rinsed with warm saline. The 3 guiding sutures were removed and the aorta closed using running sutures in a standard fashion. Transesophageal echocardiography was performed after the patient was weaned from cardiopulmonary bypass, to assess correct implantation of the prosthesis and to detect the presence of any valve leakage.

\section{Statistical Analysis}

Continuous data are presented as mean and standard deviation, or median and interquartile range; categorical data are presented as frequency and percentage. Comparisons between groups were made using the $t$ test, the Wilcoxon signed-rank test, the Mann-Whitney $U$ test, $\chi^{2}$ analysis, and the Fisher exact test, as appropriate. All tests were 2-tailed. To balance the 2 groups, a propensity-score analysis was performed. We identified a prior selection of covariates presumed to be associated with the choice between using either TAVI or SU-AVR, and these were included in a multivariable logistic regression analysis, with intervention as the 
dependent variable, to determine the propensity score. ${ }^{12}$ The independent covariates selected were as follows: age, gender, diabetes, extra-cardiac arteriopathy, chronic lung disease, previous cardiac surgery, renal impairment, pulmonary hypertension, and left ventricular ejection fraction, all as defined ${ }^{13}$ for EuroSCORE II; body mass index; and history of coronary artery disease (defined as previous coronary intervention or bypass grafting at the time of intervention). In propensity-score matching, each TAVI-treated patient was matched with an SU-AVR patient with the closest propensity score, selected using a greedy algorithm. ${ }^{14}$ Goodness for matched pairs is defined as those with the least absolute difference in matched propensity score. Matching was calculated without replacement. The balance between TAVI-treated patients and matched SU-AVR-treated patients was assessed using Wilcoxon signed-rank and Mann-Whitney $U$ tests and was reported for the same number of registry patients from both before and after propensity-score matching. ${ }^{15-18}$ To ascertain whether access type transapical (TA-TAVI) and transfemoral TAVI (TF-TAVI) could have confounded the main findings, we repeated all analyses separately. Statistical analyses were conducted using SAS, version 9.3 (SAS Institute, Cary, NC).

The primary aim of this study was to evaluate the following outcomes 30 days after the index procedure (or longer if the patient was not discharged from the treatment hospital or from a secondary convalescent facility), considering the following outcomes as defined by the updated Valve Academic Research Consortium ${ }^{19}$ (VARC-2): all-cause mortality; device success; any stroke; bleeding (life-threatening or major); permanent pacemaker implantation; any myocardial infarction; and paravalvular leakage (PVL). Secondary objectives were evaluation of the following: 1-year mortality, postoperative transaortic gradients, left ventricular ejection fraction, hours of intubation, and intensive care unit as well as in-hospital length of stay.

\section{RESULTS}

The total number of patients who underwent TAVI or SU-AVR were $1885(86.6 \%)$ and $292(13.4 \%)$, respectively. The 2 cohorts had significantly different preoperative characteristics (Table 1). The patients who underwent TAVI were older $(81.7 \pm 6$ vs $76.8 \pm 5$ years, $P<.001)$ and more likely to suffer from the following conditions: extracardiac arteriopathy $(34.9 \%$ vs $17.1 \%, P<.001)$; chronic lung disease $(24.5 \%$ vs $14.0 \%, P<.001)$; renal impairment (glomerular filtration rate $<51 \mathrm{~mL} / \mathrm{min}$ in $58.5 \%$ vs $23 \%$, $P<.001)$; and severe pulmonary hypertension $(10.8 \%$ vs $0.3 \%, P<.001)$. In addition, they had a significantly worse New York Heart Association functional class (III or IV: $80.7 \%$ vs $72.3 \%, P<.001)$. As a consequence, they had a significantly higher logistic EuroSCORE $(21.1 \% \pm 13.6 \%$ vs $9.5 \% \pm 6 \%, P<.001)$. After propensity matching, we obtained the following numbers of matched pairs of patients: (1) in overall analysis TAVI versus SU-AVR, 214; (2) in transapical access versus SU-AVR, 105; and (3) in transfemoral versus SU-AVR, 206. Matched cohorts were well balanced, and no difference in any variable was found after matching (Table 2). Postmatching balancing of other baseline variables that were not included in the propensity-score analysis indicated a similar logistic EuroSCORE for matched TAVI versus SU-AVR cohorts $(12.4 \% \pm 9.1 \%$ vs $10.5 \% \pm 6.2 \%$, $P=.09$; Table 3). Table 4 and Figure 1 show outcomes
TABLE 1. Preoperative characteristics

\begin{tabular}{|c|c|c|c|}
\hline Characteristics & $\begin{array}{c}\text { SU-AVR } \\
n=292\end{array}$ & $\begin{array}{c}\text { TAVI } \\
\mathbf{n}=\mathbf{1 8 8 5}\end{array}$ & $P$ value \\
\hline Age (y) & $76.8 \pm 5$ & $81.7 \pm 6$ & $<.001$ \\
\hline Gender, female & $188(64.4)$ & $1139(60.4)$ & .19 \\
\hline Body mass index & $27.7 \pm 4.7$ & $25.8 \pm 4.5$ & $<.001$ \\
\hline Diabetes & 77 (26.4) & $483(25.6)$ & .78 \\
\hline Arterial hypertension & $263(90.1)$ & $1537(81.5)$ & $<.001$ \\
\hline Extracardiac arteriopathy* & $50(17.1)$ & $657(34.9)$ & $<.001$ \\
\hline Chronic lung disease* & $41(14.0)$ & $462(24.5)$ & $<.001$ \\
\hline Previous cardiac surgery* & $29(9.9)$ & $348(18.5)$ & $<.001$ \\
\hline \multicolumn{4}{|l|}{ Renal impairment (mL/min)* } \\
\hline$>85$ & $107(36.6)$ & $97(5.2)$ & \\
\hline $51-85$ & $117(40.1)$ & $618(33.2)$ & $<.001$ \\
\hline$<51$ & $67(23)$ & $1090(58.5)$ & \\
\hline Dialysis & $1(0.3)$ & $59(3.2)$ & \\
\hline \multicolumn{4}{|l|}{ Pulmonary hypertension* $(\mathrm{mm} \mathrm{Hg})$} \\
\hline No & $246(84.3)$ & $742(39.4)$ & $<.001$ \\
\hline $31-55$ & $45(15.4)$ & $939(49.8)$ & \\
\hline$>55$ & $1(0.3)$ & $204(10.8)$ & \\
\hline History of coronary artery disease $\dagger$ & $12(4.1)$ & 767 (40.7) & $<.001$ \\
\hline Left ventricular ejection fraction (\%) & $58.4 \pm 9.0$ & $53.6 \pm 12.3$ & $<.001$ \\
\hline Neurological dysfunction $\ddagger$ & 0 & $170(9.0)$ & $<.001$ \\
\hline Logistic EuroSCORE $\ddagger$ & $9.5 \pm 6$ & $21.1 \pm 13.6$ & $<.001$ \\
\hline Aortic valve area $\left(\mathrm{cm}^{2}\right)$ & $0.45 \pm 0.16$ & $0.46 \pm 0.14$ & .26 \\
\hline Peak aortic gradient (mm Hg) & $87.4 \pm 22.8$ & $81.3 \pm 22.5$ & $<.001$ \\
\hline Mean aortic gradient $(\mathrm{mm} \mathrm{Hg})$ & $52.3 \pm 15.1$ & $50.3 \pm 15.0$ & .05 \\
\hline NYHA functional classification & & & $<.001$ \\
\hline I & $6(2.1)$ & $50(2.7)$ & \\
\hline II & $75(25.7)$ & 315 (16.7) & \\
\hline III & $192(65.8)$ & $1285(68.2)$ & \\
\hline IV & $19(6.5)$ & $235(12.5)$ & \\
\hline
\end{tabular}

Only variables with $<1 \%$ missing values were included in the analysis. $S U-A V R$, Sutureless aortic valve replacement; TAVI, transcatheter aortic valve implantation; EuroSCORE, European system for cardiac operative risk evaluation; NYHA, New York Heart Association. *Per EuroSCORE II definition. †Previous coronary artery bypass grafting or percutaneous transluminal coronary angioplasty \pm stenting and/or coronary disease (per Society of Thoracic Surgeons definition) at the moment of intervention. †Per EuroSCORE definition.

and the size effect, according to the VARC- $2,{ }^{19}$ before and after matching. In particular, Figure 1 shows the impact on outcomes of the 2 procedures and of the 2 different TAVI accesses. Table 5 depicts postoperative gradients, left ventricular ejection fraction, intubation time, and length of stay, before and after matching.

\section{Unmatched TAVI Versus SU-AVR}

Before matching, patients who underwent TAVI had a significantly higher 30-day mortality than did those who underwent SU-AVR ( $7 \%$ vs $2 \%, P=.001)$. In addition, 1 -year mortality was significantly higher among these patients in the unmatched group $(12.9 \%$ vs $4.6 \%$, $P<.001)$. Patients treated with TAVI had lower device success $(88.1 \%$ vs $98.6 \%, P<.001)$ and a higher incidence of life-threatening or major bleeding (20.4 vs $15.1, P=.03$ ). Severe PVL (5.2\% vs $0.3 \%, P<.001)$, as well as any PVL, 
TABLE 2. Characteristics of SU-AVR and TAVI patients before and after matching

\begin{tabular}{|c|c|c|c|}
\hline Characteristics & SU-AVR & TAVI & $P$ value \\
\hline \multicolumn{4}{|l|}{ Age $(y)$} \\
\hline Before matching* & $76.8 \pm 5$ & $81.7 \pm 6$ & $<.001$ \\
\hline \multicolumn{4}{|l|}{ After matching } \\
\hline Overall $\dagger$ & $77.4 \pm 5.4$ & $77.7 \pm 7.9$ & .07 \\
\hline $\mathrm{TA}_{\ddagger}^{\dagger}$ & $78.3 \pm 5.3$ & $78.5 \pm 8.7$ & .17 \\
\hline $\mathrm{TF} \S$ & $77.7 \pm 5.0$ & $77.7 \pm 7.0$ & .56 \\
\hline \multicolumn{4}{|l|}{ Female gender } \\
\hline Before matching* & $188(64.4)$ & $1139(60.4)$ & .19 \\
\hline \multicolumn{4}{|l|}{ After matching } \\
\hline Individual $\dagger$ & $138(64.5)$ & $139(65.0)$ & .91 \\
\hline $\mathrm{TA}_{\ddagger}^{\ddagger}$ & $69(65.7)$ & $69(65.7)$ & $>.99$ \\
\hline $\mathrm{TF} \S$ & $139(68.0)$ & $131(64.0)$ & .40 \\
\hline \multicolumn{4}{|l|}{ Body mass index } \\
\hline Before matching* & $27.7 \pm 4.7$ & $25.8 \pm 4.5$ & $<.001$ \\
\hline \multicolumn{4}{|l|}{ After matching } \\
\hline Individual $\dagger$ & $27.5 \pm 4.7$ & $27.6 \pm 5.2$ & .98 \\
\hline $\mathrm{TA} \ddagger$ & $26.9 \pm 4.6$ & $26.8 \pm 4.8$ & .76 \\
\hline $\mathrm{TF} \S$ & $27.5 \pm 4.6$ & $27.7 \pm 5.5$ & .93 \\
\hline \multicolumn{4}{|l|}{ Diabetes } \\
\hline Before matching* & 77 (26.4) & $483(25.6)$ & .78 \\
\hline \multicolumn{4}{|l|}{ After matching } \\
\hline Individual $\dagger$ & $59(27.6)$ & $58(27.1)$ & .91 \\
\hline $\mathrm{TA} \ddagger$ & $27(25.7)$ & $28(26.7)$ & .87 \\
\hline $\mathrm{TF} \S$ & $55(26.7)$ & $56(27.2)$ & .91 \\
\hline \multicolumn{4}{|c|}{ Extracardiac arteriopathy } \\
\hline Before matching* & $50(17.1)$ & $657(34.9)$ & $<.001$ \\
\hline \multicolumn{4}{|l|}{ After matching } \\
\hline Individual $\dagger$ & $46(21.5)$ & $48(22.4)$ & .81 \\
\hline $\mathrm{TA} \ddagger$ & $33(31.4)$ & 37 (35.2) & .55 \\
\hline $\mathrm{TF} \S$ & $38(18.5)$ & $38(18.5)$ & $>.99$ \\
\hline \multicolumn{4}{|l|}{ Chronic lung disease } \\
\hline Before matching* & $41(14.0)$ & $462(24.5)$ & $<.001$ \\
\hline \multicolumn{4}{|l|}{ After matching } \\
\hline Individual $\dagger$ & $39(18.2)$ & $36(16.8)$ & .70 \\
\hline $\mathrm{TA} \ddagger$ & $20(19.1)$ & $20(19.1)$ & $>.99$ \\
\hline $\mathrm{TF} \S$ & $36(17.5)$ & $38(18.5)$ & .79 \\
\hline \multicolumn{4}{|c|}{ Previous cardiac surgery } \\
\hline Before matching* & $29(9.9)$ & $348(18.5)$ & $<.001$ \\
\hline \multicolumn{4}{|l|}{ After matching } \\
\hline Individual $\dagger$ & $22(10.3)$ & $21(9.8)$ & .87 \\
\hline $\mathrm{TA} \ddagger$ & $15(14.3)$ & $12(11.4)$ & .53 \\
\hline $\mathrm{TF} \S$ & $19(9.2)$ & $22(10.7)$ & 62 \\
\hline \multicolumn{4}{|c|}{ Renal impairment (mL/min) } \\
\hline \multicolumn{4}{|l|}{ Before matching* } \\
\hline$>85$ & $107(36.6)$ & $97(5.2)$ & \\
\hline $51-85$ & $117(40.1)$ & $618(33.2)$ & $<.001$ \\
\hline$<51$ & $67(23)$ & $1090(58.5)$ & \\
\hline Dialysis & $1(0.3)$ & $59(3.2)$ & \\
\hline \multicolumn{4}{|l|}{ After matching } \\
\hline \multicolumn{4}{|l|}{ Individual $\dagger$} \\
\hline$>85$ & $46(21.5)$ & $42(19.6)$ & \\
\hline $51-85$ & $101(47.2)$ & $102(47.6)$ & .96 \\
\hline$<51$ & $66(30.8)$ & $69(32.2)$ & \\
\hline Dialysis & $1(0.5)$ & $1(0.5)$ & \\
\hline
\end{tabular}

TABLE 2. Continued

\begin{tabular}{|c|c|c|c|}
\hline Characteristics & SU-AVR & TAVI & $P$ value \\
\hline \multicolumn{4}{|l|}{$\mathrm{TA} \ddagger$} \\
\hline$>85$ & $10(9.5)$ & $12(11.4)$ & \multirow{4}{*}{.65} \\
\hline $51-85$ & 45 (42.9) & $49(46.7)$ & \\
\hline$<51$ & $49(46.7)$ & 44 (41.9) & \\
\hline Dialysis & $1(1.0)$ & 0 & \\
\hline \multicolumn{4}{|l|}{$\mathrm{TF} \S$} \\
\hline$>85$ & $45(21.8)$ & $40(19.4)$ & \multirow{4}{*}{.70} \\
\hline $51-85$ & 94 (45.6) & $104(50.5)$ & \\
\hline$<51$ & $66(32.0)$ & $60(29.1)$ & \\
\hline Dialysis & $1(0.5)$ & $2(1.0)$ & \\
\hline \multicolumn{4}{|c|}{ Pulmonary hypertension $(\mathrm{mm} \mathrm{Hg})$} \\
\hline \multicolumn{4}{|l|}{ Before matching* } \\
\hline No & $246(84.3)$ & 742 (39.4) & \multirow[t]{3}{*}{$<.001$} \\
\hline $31-55$ & $45(15.4)$ & 939 (49.8) & \\
\hline$>55$ & $1(0.3)$ & $204(10.8)$ & \\
\hline \multicolumn{4}{|l|}{ After matching } \\
\hline Individual $\uparrow$ & & & \multirow{4}{*}{.59} \\
\hline No & $168(78.5)$ & $170(79.4)$ & \\
\hline $31-55$ & 45 (21.0) & 44 (20.6) & \\
\hline$>55$ & $1(0.5)$ & $1(0.5)$ & \\
\hline \multicolumn{4}{|l|}{$\mathrm{TA} \dagger$} \\
\hline No & 71 (67.6) & 75 (71.4) & \multirow{3}{*}{.65} \\
\hline $31-55$ & $33(31.4)$ & $28(26.7)$ & \\
\hline$>55$ & $1(1.0)$ & $2(1.9)$ & \\
\hline \multicolumn{4}{|l|}{$\mathrm{TF} \S$} \\
\hline No & $160(77.7)$ & $158(76.7)$ & \multirow{3}{*}{.57} \\
\hline $31-55$ & 45 (21.8) & $48(23.3)$ & \\
\hline$>55$ & $1(0.5)$ & 0 & \\
\hline \multicolumn{4}{|l|}{ History of CAD } \\
\hline Before matching* & $12(4.1)$ & 767 (40.7) & $<.001$ \\
\hline \multicolumn{4}{|l|}{ After matching } \\
\hline Individual $\dagger$ & $12(5.6)$ & $11(5.1)$ & .83 \\
\hline $\mathrm{TA} \dagger$ & $12(11.4)$ & $15(14.3)$ & .53 \\
\hline $\mathrm{TF} \S$ & $12(5.8)$ & $15(7.3)$ & .55 \\
\hline \multicolumn{4}{|c|}{ Left ventricular ejection fraction $(\%)$} \\
\hline Before matching* & $58.4 \pm 9.0$ & $53.6 \pm 12.3$ & $<.001$ \\
\hline \multicolumn{4}{|l|}{ After matching } \\
\hline Individual $\uparrow$ & $57.7 \pm 9.3$ & $58.2 \pm 10.2$ & .41 \\
\hline $\mathrm{TA} \ddagger$ & $57.3 \pm 10.6$ & $57.3 \pm 11.2$ & .87 \\
\hline $\mathrm{TF} \S$ & $57.6 \pm 9.3$ & $57.7 \pm 9.5$ & .82 \\
\hline
\end{tabular}

Data are $\mathrm{n}(\%)$, or mean \pm standard deviation. Only variables with $<1 \%$ missing values were included in the analysis. Comparisons between groups were made using the Wilcoxon signed-rank and Mann-Whitney $U$ tests. SU-AVR, Sutureless aortic valve replacement; $T A V I$, transcatheter aortic valve implantation; $T A$, transapical; $T F$, transfemoral; $C A D$, coronary artery disease. $* 292$ patients were in the SU-AVR-treated group; 1885 were in the TAVI-treated group. $\dagger 214$ patients were in the SU-AVR-treated group; 214 were in the TAVI-treated group. $\ddagger 105$ patients were in the SU-AVR-treated group; 105 were in the TAVI-treated group. \$206 patients were in the SU-AVR-treated group; 206 patients were in the TAVI-treated group.

was higher in patients undergoing TAVI (37.3\% vs $2.0 \%$; $P<.001)$. No significant differences were found in the unmatched cohorts in stroke or myocardial infarction. A trend toward a higher pacemaker implantation rate in patients undergoing SU-AVR was found $(8.9 \%$ vs $6.2 \%$, $P=.07)$. 
TABLE 3. Preoperative variables not considered for matching

\begin{tabular}{|c|c|c|c|c|c|c|c|c|c|c|c|c|}
\hline \multirow[b]{2}{*}{ Variable } & \multicolumn{3}{|c|}{ Before matching } & \multicolumn{3}{|c|}{ After matching } & \multicolumn{3}{|c|}{ After matching } & \multicolumn{3}{|c|}{ After matching } \\
\hline & $\begin{array}{c}\text { SU-AVR } \\
(n=292)\end{array}$ & $\begin{array}{c}\text { TAVI } \\
(\mathbf{n}=\mathbf{1 8 8 5})\end{array}$ & $\begin{array}{c}P \\
\text { value }\end{array}$ & $\begin{array}{c}\text { SU-AVR } \\
(n=214)\end{array}$ & $\begin{array}{c}\text { TAVI } \\
(\mathrm{n}=\mathbf{2 1 4})\end{array}$ & $\begin{array}{c}P \\
\text { value }\end{array}$ & $\begin{array}{l}\text { SU-AVR } \\
(\mathbf{n}=\mathbf{1 0 5})\end{array}$ & $\begin{array}{c}\text { TAVI } \\
(\mathbf{n}=\mathbf{1 0 5})\end{array}$ & $\begin{array}{c}P \\
\text { value }\end{array}$ & $\begin{array}{c}\text { SU-AVR } \\
(n=206)\end{array}$ & $\begin{array}{c}\text { TAVI } \\
(\mathbf{n}=\mathbf{2 0 6})\end{array}$ & $\begin{array}{c}P \\
\text { value }\end{array}$ \\
\hline $\begin{array}{l}\text { Logistic } \\
\quad \text { EuroSCORE }\end{array}$ & $9.5 \pm 6$ & $21.1 \pm 13.6$ & $<.001$ & $10.5 \pm 6.2$ & $12.4 \pm 9.1$ & .09 & $12.6 \pm 7.2$ & $14.7 \pm 9.2$ & .11 & $10.5 \pm 6.2$ & $11.8 \pm 7.1$ & .04 \\
\hline $\begin{array}{l}\text { Functional aortic } \\
\text { valve area }\left(\mathrm{cm}^{2}\right)\end{array}$ & $0.45 \pm 0.16$ & $0.46 \pm 0.14$ & .26 & $0.43 \pm 0.14$ & $0.46 \pm 0.14$ & .12 & $0.42 \pm 0.10$ & $0.47 \pm 0.15$ & .04 & $0.44 \pm 0.15$ & $0.44 \pm 0.14$ & .99 \\
\hline $\begin{array}{c}\text { Peak aortic } \\
\text { gradient } \\
(\mathrm{mm} \mathrm{Hg})\end{array}$ & $87.4 \pm 22.8$ & $81.7 \pm 22.5$ & $<.001$ & $87.5 \pm 23.4$ & $84.5 \pm 22.5$ & .22 & $86.7 \pm 25.1$ & $80.6 \pm 20.0$ & .21 & $85.6 \pm 21.9$ & $88.2 \pm 21.9$ & .27 \\
\hline $\begin{array}{l}\text { Mean aortic } \\
\text { gradient } \\
(\mathrm{mm} \mathrm{Hg})\end{array}$ & $52.3 \pm 15.1$ & $50.3 \pm 15.0$ & .051 & $53.2 \pm 15.9$ & $52.3 \pm 15.7$ & .51 & $53.0 \pm 17.9$ & $49.7 \pm 14.1$ & .34 & $52.2 \pm 15.4$ & $54.5 \pm 17.8$ & .07 \\
\hline $\begin{array}{l}\text { Neurological } \\
\text { dysfunction } \\
\text { disease }\end{array}$ & 0 & $170(9.0)$ & $<.001$ & 0 & $21(9.8)$ & $<.01$ & 0 & $10(9.5)$ & .001 & 0 & 23 (11.0) & $<.001$ \\
\hline $\begin{array}{l}\text { Arterial } \\
\text { hypertension }\end{array}$ & $263(90.1)$ & $1537(81.5)$ & $<.001$ & $190(88.8)$ & $160(74.8)$ & $<.01$ & 93 (88.6) & $84(80.0)$ & .08 & $181(87.9)$ & $150(73.0)$ & $<.001$ \\
\hline $\begin{array}{l}\text { NYHA functional } \\
\text { class }\end{array}$ & & & $<.001$ & & & & & & & & & \\
\hline I & $6(2.1)$ & $50(2.7)$ & & $5(2.3)$ & $5(2.34)$ & & $1(0.9)$ & $2(1.9)$ & & $3(1.5)$ & $38(3.9)$ & \\
\hline II & $75(25.7)$ & 315 (16.7) & & $60(28.0)$ & $52(24.3)$ & .59 & $31(29.5)$ & $17(16.2)$ & .03 & 59 (28.6) & $50(24.3)$ & .37 \\
\hline III & $192(65.8)$ & $1285(68.2)$ & & $134(62.6)$ & $135(63.1)$ & & $66(62.0)$ & $70(66.7)$ & & $130(63.1)$ & 133 (64.6) & \\
\hline IV & $19(6.5)$ & $235(12.5)$ & & $15(7.0)$ & $22(10.3)$ & & 7 (6.7) & $16(15.2)$ & & $14(6.8)$ & $15(7.3)$ & \\
\hline
\end{tabular}

SU-AVR, Sutureless aortic valve replacement; TAVI, transcatheter aortic valve implantation; EuroSCORE, European system for cardiac operative risk evaluation; NYHA, New York Heart Association.

\section{Matched TAVI Versus SU-AVR}

After matching all patients treated with TAVI with those treated with SU-AVR, we did not observe significant differences in 30-day or 1-year mortality, stroke, bleeding, or myocardial infarction. Patients treated with TAVI had a significantly lower rate of device success $(88.8 \%$ vs $98.6 \%, P<.001)$ and pacemaker implantation $(2.8 \%$ vs $9.4 \%, P=.004)$. Severe PVL (5.1\% vs $0.5 \%)$, as well as any PVL $(35.3 \%$ vs $2.8 \%, P<.001)$, was higher in patients treated with TAVI.

\section{Matched TA-TAVI Versus SU-AVR}

Patients who underwent TA-TAVI versus SU-AVR did not show significant differences in 30-day or 1-year mortality, device success, stroke, bleeding, pacemaker implantation, myocardial infarction, or severe PVL. The only significant difference was found in mild PVL, which was significantly higher in TA-TAVI patients $(36.1 \%$ vs $2.9 \%$, $P<.001)$, but not severe PVL $(1.0 \%$ vs $1.0 \%, P>.99)$.

\section{Matched TF-TAVI Versus SU-AVR}

Patients treated with TF-TAVI, compared with SU-AVR, showed a lower rate of device success $(85.9 \%$ vs $98.1 \%$, $P<.001)$. Furthermore, in the TF-TAVI cohort, we observed a higher incidence of severe PVL $(6.3 \%$ vs $0.5 \%, P=.001)$, and any PVL $(33.5 \%$ vs $3.4 \%$, $P<.01)$. No significant differences were found between groups in 30-day or 1-year mortality, stroke, bleeding, pacemaker implantation, or myocardial infarction.

\section{Hemodynamic Outcomes and Hospital Stay}

Table 5 shows hemodynamic results and postoperative stay length in the intensive care unit for patients treated with TAVI versus SU-AVR. TAVI prostheses have significantly lower transaortic gradients in both the unmatched and matched cohorts (TAVI vs SU-AVR mean gradients: $11.0 \pm 4.6 \mathrm{~mm} \mathrm{Hg}$ vs $13.7 \pm 6.6 \mathrm{~mm} \mathrm{Hg}$, $P<.001)$. The left ventricular ejection fraction did not differ between groups. Postoperative intensive care stay, as well as in-hospital stay, was significantly longer in patients treated with SU-AVR, in both the unmatched and matched cohorts. Preoperative characteristics and outcomes of patients excluded from matching are shown in Appendixes 3 and 4 , respectively.

\section{DISCUSSION}

The main findings of this study are that TAVI and SU-AVR have similar outcomes in 30-day mortality, 1-year mortality, stroke, bleeding, and myocardial infarction. However, TAVI shows a higher incidence of postprocedural PVL and a trend toward a lower rate of permanent pacemaker implantation. Furthermore, TAVI provides lower transaortic gradients and shorter inhospital and intensive care unit stay. 
TABLE 4. Outcomes according to Valve Academic Research Consortium-2 definitions in patients treated with SU-AVR and TAVI, before and after matching

\begin{tabular}{|c|c|c|c|}
\hline Outcome & SU-AVR & TAVI & $P$ value \\
\hline \multicolumn{4}{|l|}{ 30-d Mortality } \\
\hline Before matching* & $6(2)$ & $134(7)$ & .001 \\
\hline \multicolumn{4}{|l|}{ After matching } \\
\hline Overall $\uparrow$ & $5(2.3)$ & $8(3.7)$ & .39 \\
\hline $\mathrm{TA} \ddagger$ & $3(2.9)$ & $4(3.8)$ & $>.99$ \\
\hline $\mathrm{TF} \S$ & $5(2.4)$ & $9(4.4)$ & .27 \\
\hline \multicolumn{4}{|l|}{ 1-y Mortality } \\
\hline Before matching* & $13(4.6)$ & 242 (12.9) & $<.001$ \\
\hline \multicolumn{4}{|l|}{ After matching } \\
\hline Overall $\uparrow$ & $12(5.8)$ & $20(9.4)$ & .16 \\
\hline $\mathrm{TA} \ddagger$ & $8(8.0)$ & $11(10.5)$ & .54 \\
\hline $\mathrm{TF} \S$ & $12(6.0)$ & $15(7.4)$ & .58 \\
\hline \multicolumn{4}{|l|}{ Device success } \\
\hline Before matching* & 288 (98.6) & $1661(88.1)$ & $<.001$ \\
\hline \multicolumn{4}{|l|}{ After matching } \\
\hline Individual $†$ & 211 (98.6) & $190(88.8)$ & $<.001$ \\
\hline $\mathrm{TA} \ddagger$ & $103(98.1)$ & $99(94.3)$ & .27 \\
\hline $\mathrm{TF} \S$ & $202(98.1)$ & 177 (85.9) & $<.001$ \\
\hline \multicolumn{4}{|l|}{ Any stroke } \\
\hline Before matching* & $6(2.1)$ & $52(2.8)$ & .48 \\
\hline \multicolumn{4}{|l|}{ After matching } \\
\hline Individual $†$ & $4(1.9)$ & $4(1.9)$ & $>.99$ \\
\hline $\mathrm{TA} \ddagger$ & $2(1.9)$ & $1(1.0)$ & $>.99$ \\
\hline $\mathrm{TF} \S$ & $2(1.0)$ & $3(1.5)$ & $>.99$ \\
\hline \multicolumn{4}{|c|}{ Bleeding (life-threatening or major) } \\
\hline Before matching* & $44(15.1)$ & $381(20.4)$ & .03 \\
\hline \multicolumn{4}{|l|}{ After matching } \\
\hline Individual $†$ & $40(18.7)$ & $34(16.1)$ & .48 \\
\hline $\mathrm{TA} \ddagger$ & $21(20.0)$ & $13(13.0)$ & .17 \\
\hline $\mathrm{TF} \S$ & $39(19.0)$ & $32(15.0)$ & .36 \\
\hline \multicolumn{4}{|c|}{ Pacemaker implantation } \\
\hline Before matching* & $26(8.9)$ & $116(6.2)$ & .07 \\
\hline \multicolumn{4}{|l|}{ After matching } \\
\hline Individual $\dagger$ & $20(9.4)$ & $6(2.8)$ & .0046 \\
\hline $\mathrm{TA} \ddagger$ & $10(9.5)$ & $4(3.8)$ & .09 \\
\hline $\mathrm{TF} \S$ & $19(9.2)$ & $12(5.8)$ & .19 \\
\hline \multicolumn{4}{|c|}{ Any myocardial infarction } \\
\hline Before matching* & $1(0.3)$ & $28(1.5)$ & .16 \\
\hline \multicolumn{4}{|l|}{ After matching } \\
\hline Individual $†$ & $1(0.5)$ & $2(0.9)$ & $>.99$ \\
\hline $\mathrm{TA} \ddagger$ & $1(1.0)$ & $2(1.9)$ & $>.99$ \\
\hline $\mathrm{TF}_{\S}$ & $1(0.5)$ & $1(0.5)$ & $>.99$ \\
\hline \multicolumn{4}{|c|}{ Severe paravalvular leakage } \\
\hline Before matching* & $1(0.3)$ & $98(5.2)$ & $<.001$ \\
\hline \multicolumn{4}{|l|}{ After matching } \\
\hline Individual $\dagger$ & $1(0.5)$ & $11(5.1)$ & .005 \\
\hline $\mathrm{TA} \ddagger$ & $1(1.0)$ & $1(1.0)$ & $>.99$ \\
\hline $\mathrm{TF} \S$ & $1(0.5)$ & $13(6.3)$ & .001 \\
\hline \multicolumn{4}{|l|}{ Paravalvular leakage } \\
\hline \multicolumn{4}{|l|}{ Before matching* } \\
\hline No & $286(98.0)$ & $1125(62.7)$ & \\
\hline Mild & $5(1.7)$ & $571(31.8)$ & $<.001$ \\
\hline$>$ Mild & $1(0.3)$ & $98(5.5)$ & \\
\hline
\end{tabular}

TABLE 4. Continued

\begin{tabular}{cccc}
\hline Outcome & SU-AVR & TAVI & P value \\
\hline After matching & & & \\
Individual $\dagger$ & & & \\
No & $208(97.2)$ & $134(64.7)$ & \\
Mild & $5(2.3)$ & $62(30.0)$ & $<.001$ \\
$>$ Mild & $1(0.5)$ & $11(5.3)$ & \\
TA $\ddagger$ & & & \\
No & $101(96.1)$ & $66(62.9)$ & \\
Mild & $3(2.9)$ & $38(36.1)$ & $<.001$ \\
$>$ Mild & $1(1.0)$ & $1(1.0)$ & \\
TF $\S$ & & & \\
No & $199(96.6)$ & $137(66.5)$ & \\
Mild & $5(2.4)$ & $56(27.2)$ & $<.001$ \\
$>$ Mild & $2(1.0)$ & $13(6.3)$ & \\
\hline
\end{tabular}

Comparisons between groups were made using the Wilcoxon signed-rank and MannWhitney $U$ tests. Data are expressed as n (\%). SU-AVR, Sutureless aortic valve replacement; $T A V I$, transcatheter aortic valve implantation; $T A$, transapical; $T F$, transfemoral; mis, missing. *292 patients were in the grouptreated with SU-AVR; 1885 patients were in the group treated with TAVI. $\nmid 214$ patients were in the group treated with SU-AVR; 214 patients were in the group treated with TAVI. $\ddagger 105$ patients were in the group treated with SU-AVR; 105 patients were in the group treated with TAVI. $\$ 206$ patients were in the group treated with SU-AVR; 206 patients were in the group treated with TAVI.

\section{Study Population}

These data come from 2 large multicenter experiences of TAVI and SU-AVR that globally include $>2000$ patients. These registries are based on a "real-world," "all-comers" enrollment, and looking at the baseline characteristics of the 2 cohorts provides clear evidence that these procedures were performed in 2 different populations. In fact, the patients who underwent TAVI were older and had significantly worse baseline characteristics, resulting in a logistic EuroSCORE that is almost double that of patients treated with SU-AVR. Consequently, the higher mortality before matching of patients treated with TAVI reflects these preoperative differences. After matching, the logistic EuroSCORE of patients in the TAVI groups was significantly reduced, whereas that of patients in the SU-AVR group remained unchanged (logistic EuroSCORE after matching: TAVI: $12.4 \% \pm 9.1 \%$; SU-AVR: $10.5 \% \pm 6.2 \%, P=.09)$. This difference means that the matching process selected, among the TAVI cohort, those patients who had the lowest preoperative risk profile. Although these patients still have an intermediate- to high-risk profile, they do not have a very high/prohibitive surgical risk and belong to the so-called "gray zone" 20,21 that identifies a patient population that may be well treated with AVR, SU-AVR, or TAVI. For this reason, a difference in 30-day mortality was not observed. Strict definition of a gray zone is difficult, as no specific guidelines are available for TAVI and SU-AVR; consequently, any assertion would be arbitrary.

The PARTNER 2 trial (Placement of Aortic Transcatheter Valve) considers intermediate risk to be indicated by a Society of Thoracic Surgeons score between $4 \%$ and 


\section{Outcomes according to procedures Odds Ratio and $95 \% \mathrm{CL}$}

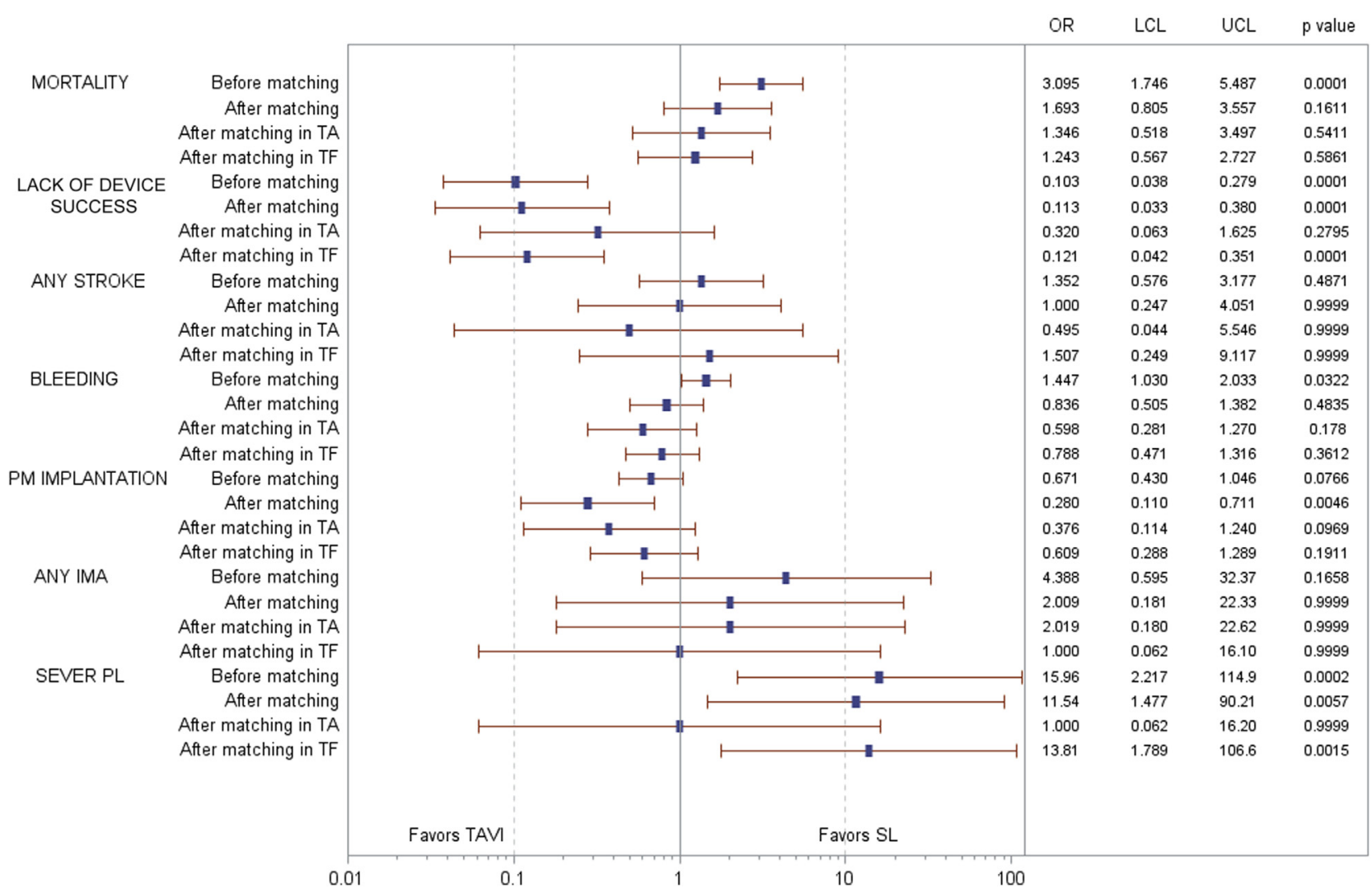

FIGURE 1. Forest plot showing outcomes according to procedure (TAVI and sutureless) and the TAVI access approach. $C L$, Confidence limit; $O R$, odds ratio; $L C L$, lower confidence limit; $U C L$, upper confidence limit; $T A$, transapical; $T F$, transfemoral; $P M$, pacemaker; $I M A$, acute myocardial infarction; $P L$, paravalvular leakage; TAVI, transcatheter aortic valve implantation; $S L$, sutureless.

$8 \%$, but since this score is not widely used in Europe, this measure is not applicable to our study population. Many reasons may lead to a patient in the "gray zone" undergoing TAVI rather than SU-AVR or conventional surgery: patient preference, surgeon preference, center policy, and in a low number of patients, anatomy. In a recent paper by Makkar and colleagues, ${ }^{22}$ with data from the PARTNER trial, only $23 \%$ of patients underwent TAVI for technical inoperability due to porcelain aorta $(40 \%)$, mediastinal radiation, chest wall deformity, or previous surgery (especially coronary artery bypass grafting). However, with the exclusion of porcelain aorta and severe chest wall deformity, which are the only absolute contraindications for SU-AVR and conventional surgery, the other anatomical conditions increase the risk of a "surgical" approach but do not actually contraindicate it; therefore, these patients could be included in a special category of "anatomical gray zone."

\section{Outcomes}

Mortality. In the TAVI versus SU-AVR analysis, mortality in patients treated with SU-AVR was $2.3 \%$, a value similar to those previously published..$^{23,24}$ In the group treated with TAVI, mortality was $3.7 \%$, much lower than that reported in previous TAVI series, ${ }^{25,26}$ but similar to mortality of other series with intermediate-risk TAVI patients. ${ }^{27,28}$ Thus, TAVI, in the "gray zone" area, seems to provide results similar to not only SU-AVR but also AVR, confirming the findings of a previously published propensity-matched study comparing AVR, TA-TAVI, and SU-AVR. ${ }^{20}$

Another important aspect to discuss is related to the TAVI approach. TAVI is basically performed through a transapical or a transfemoral access approach, which have significant differences. TA-TAVI requires general anesthesia, intubation, and a surgical incision, whereas TF-TAVI may be performed easily in a totally percutaneous fashion on an awake patient using just sedation. However, given that the length of the transapical delivery system is shorter than that of the transfemoral, the deployment of the valve during TA-TAVI may be more precise than deployment in TF-TAVI. Although these 2 approaches have not been clearly demonstrated to have significantly different outcomes, ${ }^{29-31}$ we chose to match patients treated with SU-AVR with the entire TAVI cohort, but 
TABLE 5. Postoperative data before and after matching

\begin{tabular}{|c|c|c|c|c|c|c|c|c|c|c|c|c|}
\hline \multirow[b]{2}{*}{ Variable } & \multicolumn{3}{|c|}{ Before matching } & \multicolumn{9}{|c|}{ After matching } \\
\hline & $\begin{array}{l}\text { SU-AVR } \\
(292)\end{array}$ & $\begin{array}{r}\text { TAVI } \\
(\mathbf{1 8 8 5}) \\
\end{array}$ & $\begin{array}{c}P \\
\text { value }\end{array}$ & $\begin{array}{l}\text { SU-AVR } \\
(214)\end{array}$ & $\begin{array}{l}\text { TAVI } \\
(214)\end{array}$ & $\begin{array}{c}P \\
\text { value }\end{array}$ & $\begin{array}{l}\text { SU-AVR } \\
(105)\end{array}$ & $\begin{array}{l}\text { TAVI } \\
(105)\end{array}$ & $\begin{array}{c}P \\
\text { value }\end{array}$ & $\begin{array}{l}\text { SU-AVR } \\
(206)\end{array}$ & $\begin{array}{l}\text { TAVI } \\
\text { (206) }\end{array}$ & $\begin{array}{c}P \\
\text { value }\end{array}$ \\
\hline $\begin{array}{l}\text { Peak aortic } \\
\text { gradient } \\
(\mathrm{mm} \mathrm{Hg})\end{array}$ & $27.2 \pm 11.7$ & $20.0 \pm 7.8$ & $<.001$ & $26.7 \pm 12.1$ & $20.3 \pm 8.1$ & $<.001$ & $26.0 \pm 10.3$ & $18.0 \pm 6.9$ & $<.001$ & $26.7 \pm 11.8$ & $20.8 \pm 8.7$ & $<.01$ \\
\hline $\begin{array}{l}\text { Mean aortic } \\
\text { gradient } \\
\text { (mm Hg) }\end{array}$ & $13.8 \pm 6.3$ & $10.8 \pm 4.5$ & $<.001$ & $13.7 \pm 6.6$ & $11.0 \pm 4.6$ & $<.001$ & $13.2 \pm 5.5$ & $10.3 \pm 4.2$ & $<.001$ & $13.6 \pm 6.4$ & $11.3 \pm 4.8$ & .001 \\
\hline $\begin{array}{l}\text { Left ventricular } \\
\text { ejection } \\
\text { fraction }(\%)\end{array}$ & $56.5 \pm 7.9$ & $54.5 \pm 11.1$ & .22 & $56.8 \pm 7.8$ & $57.5 \pm 8.6$ & .41 & $56.5 \pm 7.3$ & $56.6 \pm 9.7$ & .80 & $55.9 \pm 7.4$ & $57.1 \pm 9.5$ & .08 \\
\hline $\begin{array}{l}\text { Hours of intubation } \\
\text { since the end of } \\
\text { the procedure }\end{array}$ & 18 [8-30] & $2[0-10]$ & $<.001$ & 18 [8-36] & $2[0-7]$ & $<.001$ & 22 [8-46] & 6 [2-13] & $<.001$ & 19 [8-36] & $0[0-4]$ & $<.001$ \\
\hline $\begin{array}{l}\text { Postoperative ICU } \\
\text { stay (d) }\end{array}$ & $2[1-3]$ & $1[1-3]$ & $<.001$ & $2[1-3]$ & $1[1-3]$ & $<.001$ & $2[1-3]$ & $2[1-3]$ & $<.001$ & $2[1-3]$ & $1[0-1]$ & $<.001$ \\
\hline $\begin{array}{l}\text { Postoperative } \\
\text { hospitalization (d) }\end{array}$ & 11 [8-15] & $7[5-11]$ & $<.001$ & 11 [8-16] & 7 [5-9] & $<.001$ & 11 [8-17] & 8 [6-9] & $<.0001$ & 11 [8-17] & $6[5-8]$ & $<.001$ \\
\hline
\end{tabular}

also separately with TA-TAVI or TF-TAVI patients, to avoid the bias related to the access approach.

Device success and paravalvular leakage. Device success is a composite endpoint defined by the Valve Academic Research Consortium as absence of procedural mortality and correct positioning of a single valve in the proper anatomical location, and good performance of the valve prosthesis (including no moderate or severe PVL and a mean gradient $<20 \mathrm{~mm} \mathrm{Hg}$ ). We observed a significantly lower rate of device success in patients who underwent TAVI than in those who underwent SU-AVR, owing mainly to the not-negligible incidence of moderate and severe PVL, and to the need for a second valve when the first is wrongly positioned in the cohort treated with TAVI. Although the lower rate of device success is present in the entire TAVI cohort and the TF-TAVI cohort, in patients treated with TA-TAVI, device success is similar to that for patients treated with SU-AVR. This finding is confirmed by the similar incidence of severe and moderate PVL in TA-TAVI and in SU-AVR.

These findings are probably due to better stability and precision during transapical deployment. Blackstone and colleagues $^{31}$ have recently reported a significantly lower incidence of PVL in patients treated with TA-TAVI than in those treated with TF-TAVI, using propensity-matched data from the PARTNER trial. Given that PVL has been directly associated with late mortality, ${ }^{32,33}$ ) this aspect of the condition should be carefully considered, especially in patients belonging to the "gray zone," who have several therapeutic options. However, the introduction of newgeneration TAVI devices that are specifically designed to increase sealing between the aortic annulus and the TAVI valve is likely to significantly decrease the incidence of PVL after TAVI. A recent report ${ }^{34}$ suggest that the new Sapien-3 valve significantly reduces PVL, compared with the Sapien-XT. Therefore, in the future, the "gap" between TAVI and SU-AVR in terms of PVL is likely to be reduced. Permanent pacemaker implantation. The incidence of permanent pacemaker implantation was higher in patients who received a sutureless bioprosthesis, and although statistical significance was reached in only the SU-AVR versus all-TAVI analysis $(P<.01)$, a trend is visible in the other groups. In fact, before matching and after matching for TA-TAVI and TF-TAVI, the pacemaker implantation rate was always higher, although not statistically significant, in patients undergoing SU-AVR. Other authors found similar results in a propensity-matched analysis of TAVI versus SU-AVR. ${ }^{35,36}$ The higher rate of pacemaker implantation in the SU-AVR group may be due to the self-expandable nature of the devices, which leads to persistent compression and to damage of the atrioventricular conduction system and left bundle branch block. A recent report with data from the Perceval European Multicenter Study (Cavalier trial) ${ }^{37}$ shows that the incidence of complete atrioventricular block, in patients with no preoperative cardiac rhythm abnormalities undergoing isolated SU-AVR, was $7.5 \%$, consistent with the rate of pacemaker implantation observed in our study. The transcatheter valve used in this study is a balloonexpandable device with a low profile, which during and after deployment, usually does not interfere with the membranous septum or the conduction system of the heart. However, a high pacemaker implantation rate has been described for self-expandable TAVI valves, ${ }^{38,39}$; 
consequently, this outcome should be evaluated carefully when choosing among various treatment strategies.

Hemodynamics. Predischarge hemodynamic data show significantly lower mean and peak gradients in the TAVI group, but the real clinical impact of this finding is unknown and should be investigated further. Transaortic gradients, especially if measured early after intervention, might be influenced by the difference in surgical technique between TAVI and SU-AVR. Postoperative anemia, hemodilution, and inflammation may have a role in the increased predischarge gradients found in the SU-AVR group.

The significantly shorter intubation time and length of stay, both in the intensive care unit and the hospital, of TAVI may be easily explained, by the less-invasive nature of these procedures, in particular the absence of extracorporeal circulation. However, the impact of the shorter length of stay on patient early and late outcomes, as well as on cost effectiveness, should be investigated further. In addition, hospital stay may vary among centers, owing to differences in policies, independent of patients' postoperative course; some centers perform rehabilitation during the same hospital stay in which the procedure is performed (resulting in a longer stay), and some centers do not send patients to rehabilitation facilities and consequently discharge patients later to monitor possible late complications that are not infrequently found in such delicate patients.

Therefore, during the evaluation process of patients who have severe symptomatic aortic valve stenosis that belong to the so-called "gray-zone," several factors should be considered carefully: rate of device success, rate of pacemaker implantation, incidence and severity of PVL, hemodynamic performance, and postoperative length of stay. Combining all these variables might help the multidisciplinary "heart team" select the most appropriate treatment for each individual patient.

\section{Study Limitations}

The main limitations of this study relate to the multicenter nature of the 2 databases from which the data originated. Not all institutions performed both procedures. Adverse events were self-adjudicated, and we did not have an echocardiographic core lab.

\section{CONCLUSIONS}

As therapeutic strategies, TAVI and SU-AVR are both reasonable in patients who have severe symptomatic aortic valve stenosis. After matching, we did not observe differences in 30-day or 1-year mortality, stroke, bleeding, or myocardial infarction. Patients treated with TAVI are more likely to show less device success and more postoperative PVL, even if this is less evident with TA-TAVI. On the other hand, SU-AVR seems to provide higher transaortic gradients, a longer postoperative length of stay, and a trend toward a higher pacemaker implantation rate. The choice of the most appropriate treatment for patients who have aortic valve stenosis and belong in the "gray zone" should be tailored according to their clinical and anatomical characteristics, to provide the optimal solution for each individual patient.

\section{Conflict of Interest Statement}

Dr D'Onofrio has financial relationships with Edwards Lifesciences (Irvine, Calif). In particular, he has been a transapical TAVI proctor in the United States and Japan for Edwards Lifesciences during the past 2 years. Dr Cassese reports lecture fees from Edwards and Medtronic. Dr Tarantini reports lecture fees from Edwards. Dr Mignosa reports lecture fees from Sorin. Dr Meuris reports lecture fees from Sorin. Dr Colombo reports equity ownership in Direct Flow. Dr Rinaldi reports lecture fees from Edwards and Medtronic. Dr Stefàno reports consulting fees from St. Jude Medical. All other authors have nothing to disclose with regard to commercial support.

You can watch a Webcast of this AATS meeting presentation by going to: http://webcast.aats.org/2015/Video/ Wednesday/04-29-15_6B_0731_D_Onofrio.mp4.

\section{References}

1. Kapadia SR, Leon MB, Makkar RR, Tuzcu EM, Svensson LG, Kodali S, et al 5-year outcomes of transcatheter aortic valve replacement compared with standard treatment for patients with inoperable aortic stenosis (PARTNER 1) a randomised controlled trial. Lancet. 2015;385:2485-91.

2. Toggweiler S, Humphries KH, Lee M, Binder RK, Moss RR, Freeman M, et al. 5-year outcome after transcatheter aortic valve implantation. J Am Coll Cardiol. 2013;61:413-9.

3. D’Onofrio A, Salizzoni S, Agrifoglio M, Cota L, Luzi G, Tartara PM, et al. Medium term outcomes of transapical aortic valve implantation: results from the Italian Registry of Trans-Apical Aortic Valve Implantation. Ann Thorac Surg. 2013;96:830-5.

4. Nishimura RA, Otto CM, Bonow RO, Carabello BA, Erwin JP III, Guyton RA, et al. 2014 AHA/ACC guideline for the management of patients with valvular heart disease: a report of the American College of Cardiology/American Heart Association Task Force on Practice Guidelines. J Thorac Cardiovasc Surg. 2014;148:e1-132.

5. Cribier A, Durand E, Eltchaninoff H. Patient selection for TAVI in 2014: Is i justified to treat low- or intermediate-risk patients? The cardiologist's view EuroIntervention. 2014;10(Suppl U):U16-21.

6. Kappetein AP, Osnabrugge RL, Head SJ. Patient selection for TAVI in 2014: Is there a justification for treating low- or intermediate-risk patients? The surgeon's view. EuroIntervention. 2014;10(Suppl U):U11-5.

7. Haverich A, Wahlers TC, Borger MA, Shrestha M, Kocher AA, Walther T, et al Three-year hemodynamic performance, left ventricular mass regression, and prosthetic-patient mismatch after rapid deployment aortic valve replacement in 287 patients. J Thorac Cardiovasc Surg. 2014;148:2854-60.

8. Folliguet TA, Laborde F, Zannis K, Ghorayeb G, Haverich A, Shrestha M. Sutureless Perceval aortic valve replacement: results of two European centers Ann Thorac Surg. 2012;93:1483-8.

9. Phan K, Tsai YC, Niranjan N, Bouchard D, Carrel TP, Dapunt OE, et al Sutureless aortic valve replacement: a systematic review and meta-analysis. Ann Cardiothorac Surg. 2015;4:100-11.

10. Schlömicher M, Haldenwang PL, Moustafine V, Bechtel M, Strauch JT. Minimal access rapid deployment aortic valve replacement: initial single-center experience and 12-month outcomes. J Thorac Cardiovasc Surg. 2015;149:434-40.

11. Gilmanov D, Miceli A, Ferrarini M, Farneti P, Murzi M, Solinas M, et al Aortic valve replacement through right anterior minithoracotomy: Can 
sutureless technology improve clinical outcomes? Ann Thorac Surg. 2014;98: 1585-92.

12. Rubin DB. Estimating causal effects from large data sets using propensity scores. Ann of Intern Med. 1997;127:757-63.

13. Nashef SA, Roques F, Sharples LD, Nillson J, Smith C, Goldstone AR, et al. EuroSCORE II. Eur J Cardiothorac Surg. 2012;41:734-44.

14. Parsons LS. Reducing bias in a propensity score matched-pair sample using greedy matching techniques. Paper 214-26. In: Proceedings of the 26th Annual SAS Users Group International Conference, Long Beach, California, April 22. 25, 2001. Cary, NC: SAS Institute; 2001. Available at: http://www2.sas.com/ proceedings/sugi26/p214-26.pdf.

15. D'Agostino RB. Propensity score methods for bias reduction in the comparison of a treatment to a non-randomized control group. Stat Med. 1998; 17:2265-81.

16. Rosenbaum PR. Optimal matching for observational studies. J Am Stat Assoc. 1989;84:1024-32.

17. Rosenbaum PR, Ruben D. The bias due to incomplete matching. Biometrics. 1985;41:103-16.

18. Rosenbaum PR, Ruben D. The central role of propensity score in observational studies for causal effects. Biometrika. 1983;70:41-55.

19. Kappetein AP, Head SJ, Généreux P, Piazza N, van Mieghem NM, Blackstone EH, et al. Updated standardized endpoint definitions for transcatheter aortic valve implantation: the Valve Academic Research Consortium-2 consensus document. J Thorac Cardiovasc Surg. 2013;145:6-23.

20. D’Onofrio A, Messina A, Lorusso R, Alfieri OR, Fusari M, Rubino P, et al. Sutureless aortic valve replacement as an alternative treatment for patients belonging to the "gray zone" between transcatheter aortic valve implantation and conventional surgery: a propensity-matched, multicenter analysis. $J$ Thorac Cardiovasc Surg. 2012;144:1010-6.

21. Muneretto C, Bisleri G, Moggi A, Di Bacco L, Tespili M, Repossini A, et al. Treating the patients in the 'grey-zone' with aortic valve disease: a comparison among conventional surgery, sutureless valves and transcatheter aortic valve replacement. Interact Cardiovasc Thorac Surg. 2015;20:90-5.

22. Makkar RR, Jilaihawi H, Mack M, Chakravarty T, Cohen DJ, Cheng W, et al. Stratification of outcomes after transcatheter aortic valve replacement according to surgical inoperability for technical versus clinical reasons. J Am Coll Cardiol. 2014;63:901-11.

23. Shrestha M, Fischlein T, Meuris B, Flameng W, Carrel T, Madonna F, et al. European multicentre experience with the sutureless Perceval valve: clinical and haemodynamic outcomes up to 5 years in over 700 patients. Eur J Cardiothorac Surg. 2016;49:234-41.

24. Miceli A, Santarpino G, Pfeiffer S, Murzi M, Gilmanov D, Concistré G, et al. Minimally invasive aortic valve replacement with Perceval S sutureless valve: early outcomes and one-year survival from two European centers. $J$ Thorac Cardiovasc Surg. 2014; 148:2838-43.

25. Thomas M, Schymik G, Walther T, Himbert D, Lefèvre T, Treede H, et al. Thirty-day results of the SAPIEN aortic Bioprosthesis European Outcome (SOURCE) Registry: a European registry of transcatheter aortic valve implantation using the Edwards SAPIEN valve. Circulation. 2010;122: 62-9.

26. Giordana F, D'Ascenzo F, Nijhoff F, Moretti C, D'Amico M, Biondi Zoccai G, et al. Meta-analysis of predictors of all-cause mortality after transcatheter aortic valve implantation. Am J Cardiol. 2014;114:1447-55.

27. D'Errigo P, Barbanti M, Ranucci M, Onorati F, Covello RD, Rosato S, et al. Transcatheter aortic valve implantation versus surgical aortic valve replacement for severe aortic stenosis: results from an intermediate risk propensitymatched population of the Italian OBSERVANT study. Int J Cardiol. 2013;167: 1945-52.

28. Wenaweser P, Stortecky S, Schwander S, Heg D, Huber C, Pilgrim T, et al. Clinical outcomes of patients with estimated low or intermediate surgical risk undergoing transcatheter aortic valve implantation. Eur Heart J. 2013;34: 1894-905.

29. Murarka S, Lazkani M, Neihaus M, Boggess M, Morris M, Gellert G, et al. Comparison of 30-day outcomes of transfemoral versus transapical approach for transcatheter aortic valve replacement: a single-center US experience. Ann Thorac Surg. 2015;99:1539-44.

30. Panchal HB, Ladia V, Amin P, Patel P, Veeranki SP, Albalbissi K, et al. A metaanalysis of mortality and major adverse cardiovascular and cerebrovascular events in patients undergoing transfemoral versus transapical transcatheter aortic valve implantation using Edwards valve for severe aortic stenosis. Am J Cardiol. 2014;114:1882-90
31. Blackstone EH, Suri RM, Rajeswaran J, Babaliaros V, Douglas PS, Fearon WF, et al. Propensity-matched comparisons of clinical outcomes after transapical or transfemoral TAVI: a PARTNER-I trial substudy. Circulation. 2015;131:1989-2000.

32. Kodali SK, Williams MR, Smith CR, Svensson LG, Webb JG, Makkar RR, et al. Two-year outcomes after transcatheter or surgical aortic-valve replacement. $N$ Engl J Med. 2012;366:1686-95.

33. Athappan G, Patvardhan E, Tuzcu EM, Svensson LG, Lemos PA, Fraccaro C, et al. Incidence, predictors, and outcomes of aortic regurgitation after transcatheter aortic valve replacement: meta-analysis and systematic review of literature. J Am Coll Cardiol. 2013;61:1585-95.

34. Nijhoff F, Abawi M, Agostoni P, Ramjankhan FZ, Doevendans PA, Stella PR. Transcatheter aortic valve implantation with the new balloon-expandable Sapien 3 versus Sapien XT valve system: a propensity score-matched single-center comparison. Circ Cardiovasc Interv. 2015;8:e002408.

35. Santarpino G, Pfeiffer S, Jessl J, Dell'Aquila AM, Pollari F, Pauschinger M, et al. Sutureless replacement versus transcatheter valve implantation in aortic valve stenosis: a propensity-matched analysis of 2 strategies in high-risk patients. $J$ Thorac Cardiovasc Surg. 2014;147:561-7.

36. D’Onofrio A, Rizzoli G, Messina A, Alfieri O, Lorusso R, Salizzoni S, et al. Conventional surgery, sutureless valves, and transapical aortic valve replacement: What is the best option for patients with aortic valve stenosis? A multicenter, propensity-matched analysis. J Thorac Cardiovasc Surg. 2013; 146:1065-70.

37. Laborde F, Fischlein T, Hakim-Meibodi K, Misfeld M, Carrel T, Zembala M, et al. Clinical and haemodynamic outcomes in 658 patients receiving the Perceval sutureless aortic valve: early results from a prospective European multicentre study (the Cavalier Trial). Eur J Cardiothorac Surg. 2016;49:978-86.

38. Adams DH, Popma JJ, Reardon MJ, Yakubov SJ, Coselli JS, Deeb GM, et al. Transcatheter aortic-valve replacement with a self-expanding prosthesis. N Engl J Med. 2014;370:1790-8.

39. Salizzoni S, Anselmino M, Fornengo C, Giordana F, La Torre M, Moretti C, et al. One-year follow-up of conduction disturbances following transcatheter aortic valve implantation. J Cardiovasc Med (Hagerstown). 2015;16:296-302.

Key Words: aortic valve replacement, heart valve replacement, transapical, percutaneous, heart valve replacement sutureless

\section{Discussion}

Dr H. Maniar (St. Louis, Mo). I would like to thank the Association for the opportunity to discuss this paper. It is a very interesting study in that it compares two real world registries from Europe, both on TAVR, particularly the balloon-expandable systems, as well as our new sutureless aortic valves.

I have several questions for you, mostly based on both methodology and then the implications that we take from your study. With regards to methodology, if you could address - and thank you for sending me the manuscriptthe reason the study intervals for the two groups were different. In the transcatheter valve population, the study interval was from 2007 to 2012, whereas in the sutureless valve group, it was from 2010 to 2014 . Are the TAVR results that we have seen here today then likely to be affected by the fact that this was probably an early-generation SAPIEN system; and was it a conscious effort, either because of logistics or conceptual reasons, that you did not use any self-expanding valves in this study? 
Dr D'Onofrio. The study periods were different because this was a retrospective registry, and we specifically asked all centers to send us data of patients with at least 1 year of follow-up. Since this registry started in 2013, the enrolled patients are only from 2007 to 2012 . For the same reason, the SAPIEN 3 valve, which is the new generation that has been demonstrated to reduce significantly the incidence of paravalvular- leaks, was not included.

There is a logistic reason why we included only transcatheter balloon-expandable valves. We decided to do this on purpose, because there is already the Italian CoreValve registry, and it would have been difficult to share these data.

Dr Maniar. In the manuscript, you allude to the fact that even after propensity matching, looking at the "healthier" TAVR patients for whom there might be an advantage to using sutureless aortic valves, particularly given the higher incidence of PVL in the TAVR cohort, this still looks like a relatively sick group of patients. They still stay in the hospital a week after the procedure.

So this leads to the question-if you can identify the healthier group of patients who underwent TAVR-of what characteristics you think of now that would be found in this gray-zone patient who you may want to pursue with sutureless aortic valve therapy, as opposed to transcatheter aortic valve therapy.

Dr D'Onofrio. Even if these patients are moderate to high risk, maybe more that are high risk than moderate, there are some situations in which you would not use a TAVI for anatomical reasons. For example, if you had a big chunk of calcium on the aortic annulus, you might predict some degree of paravalvular leakage after TAVI; therefore, annular decalcification with an open technique and sutureless valve implantation should be preferred. In case of a very small aortic annulus, sutureless valves could generate a patient-prosthesis mismatch, and consequently, a TAVI valve could be the most appropriate choice. In other words, there are some situations related to the specific features of each individual patient that can guide surgeons during the decision process.

In this study, we did not consider frailty scores or the eyeball test for frailty evaluation. Sometimes the decision is based on the subjective evaluation of patients' general conditions and not only considering risk scores and predictors of bad outcomes.

Dr V. Thourani (Atlanta, $\mathrm{Ga}$ ). That was a very nice presentation. Congratulations. I have a question for you. I am really surprised at the pacemaker rate; $9 \%$ for a sutureless valve seems really high to me, and $2.8 \%$ on the transcatheter side seems relatively low to me. Can you explain those results?

Dr D'Onofrio. This is the same question that we asked looking at the data. Actually, there is wide variability of pacemaker implantation rate depending on the center where the sutureless valves were implanted. We believe that there are three main reasons that explain this incidence of pacemaker implantation. The first is sutureless valve oversizing that should be avoided. Second is decalcification of the annulus that should be complete. The third is an aggressive pacemaker implantation policy. However, looking at the literature, the mean incidence of pacemaker rate in sutureless valves is almost $8 \%$ to $9 \%$. So these are the three reasons that we use to explain this difference.

Dr Thourani. In the manuscript, it would be nice maybe to even say which patients had preoperative bundle branch block, which had a higher chance for a potential postoperative pacemaker. We have done that in the TAVR population, and that has been very reliable in predicting who is going to have a pacemaker.

Dr D'Onofrio. I agree. 
APPENDIX 1. Participating study sites of the Italian Transcatheter Expandable-Balloon Registry, members of the TAVI team, and number of patients enrolled

\begin{tabular}{|c|c|c|}
\hline Institution & Members of the TAVI team & Patients \\
\hline Centro Cardiologico Monzino, Milan & M. Agrifoglio, A. Bartorelli & 358 \\
\hline San Raffaele Hospital, Milan & A. Chieffo, A. Colombo, G. Giustino, O. Alfieri, M. Montorfano & 248 \\
\hline University of Padua & G. Gerosa, A. D’Onofrio, C. Tessari, G. Tarantini, V. Gasparetto, L. Besola & 184 \\
\hline IRCCS San Martino-IST, Genova & T. Regesta, F. Repetto, G. Passerone, V. Boasi, M. Vischi & 126 \\
\hline Sant'Anna, Catanzaro & M. Cassese, ${ }^{*}$ G. Martinelli,* B. Missiroli & 122 \\
\hline University of Turin & M. Rinaldi, S. Salizzoni, M. La Torre, M. D’Amico, C. Moretti, M. Lupo & 80 \\
\hline Hesperia Hospital, Modena & D. Gabbieri, A. Benassi, G. D’Annibale & 77 \\
\hline AOU S. Orsola, Bologna & F. Saia, C. Savini, A. Marzocchi, R. Di Bartolomeo & 72 \\
\hline Ferrarotto Hospital, Catania & C. Tamburino, S. Immè, C. Sgroi, AS. Rubino & 71 \\
\hline AOU Integrata Verona & F. Ribichini, G. Faggian, F. Onorati & 59 \\
\hline AO Papa Giovanni XXIII, Bergamo & D. Cugola, A. Terzi, O. Valsecchi & 52 \\
\hline IRCCS Policlinico S. Matteo, Pavia & M. Aiello, M. Ferrario & 49 \\
\hline AO Brotzu, Cagliari & E.M. Cirio, G. Lixi, F. Sanna, A. Boi, B. Loi & 42 \\
\hline AOU Policlinico Le Scotte, Siena & A. Iadanza, C. Pierli, G. Sincropi & 36 \\
\hline AOU S. Maria Misericordia, Udine & U. Livi, E. Pompei, E. Mazzaro, A. Proclemer, L. Spedicato, G. Bernardi & 34 \\
\hline AOU Careggi, Firenze & P. Stefàno, M. Stolcova, G. Santoro & 33 \\
\hline Humanitas Research Hospital, Rozzano & D. Ornaghi, A. Cappai & 28 \\
\hline Ospedale Cattinara, Trieste & A. Minati, A. Salvi, A. Pappalardo & 22 \\
\hline Ospedale Maggiore, Parma & A. Agostinelli, D. Ardissino, T. Gherli & 22 \\
\hline Clinica S. Maria, Bari & M. Cassese, ${ }^{*}$ G. Martinelli* & 20 \\
\hline AO San Camillo-Forlanini, Roma & F. Musumeci, R. Violini, C. Boschetti, R. Fiorilli, G.Luzi, V. Polizzi & 20 \\
\hline AO Cesare Biagio e Arrigo, Alessandria & A. Audo, C. Cavozza, M. Reale, G. Ballestrero & 18 \\
\hline San Donato, Milano & F. Casilli, L. Inglese, C. De Vincentis, A. Garatti & 17 \\
\hline Instituto Clinico Sant'Ambrogio, Milano & F. Bedogni, N. Brambilla, P. Panisi & 16 \\
\hline AOUP Federico II, Napoli & R.A. Mozzillo, V. De Amicics, C. Vosa, C. Rapacciuolo & 14 \\
\hline AOU Pisana, Pisa & S. Petronio, E. Giannini, A. Pieroni, G. Scioti & 14 \\
\hline Ospedale S. Chiara, Trento & M. Dallago, R. Bonmassari, A. Graffigna & 12 \\
\hline Clinica Mediterranea, Napoli & C. Briguori, G. Di Maio, L. Marino & 11 \\
\hline Città di Lecce, GVM Care \& Research & A. Liso, R. Gregorini & 10 \\
\hline Casa di cura Columbus, Milano & A. Colombo & 9 \\
\hline Niguarda, Milano & G. Bruschi, L. Martinelli, F. De Marco, S. Klugman & 4 \\
\hline Policlinico Umberto I, Roma & F. Miraldi, G. Sardella & 4 \\
\hline Spedali Civili, Brescia & C. Fiorina, F. Ettori & 1 \\
\hline
\end{tabular}

*M. Cassese and G. Martinelli moved to Bari in July 2012.

APPENDIX 2. Partecipating study sites of the Sutureless European multicenter study group, members of the surgical team, and number of patients enrolled

\begin{tabular}{llr}
\hline \multicolumn{1}{c}{ Institution } & \multicolumn{1}{c}{ Members of the surgical team } & Patients \\
\hline Klinikum Nürnberg, Nuremberg, Germany & T. Fischlein, G. Santarpino, F. Pollari, F. Vogt & 167 \\
AOU Policlinico Vittorio Emanuele, University of Catania & C. Mignosa, A.S. Rubino & 56 \\
Karolinska Institutet and University Hospital, Stockholm & M. Dalén, P. Svenarud, U. Sartipy & 24 \\
Ospedali Riuniti, Trieste & A. Pappalardo, G. Gatti, B. Benussi & 19 \\
Oulu University Hospital, Oulu, Finland & F. Biancari & 16 \\
University Hospital Gasthuisberg, Leuven, Belgium & B. Meuris & 12 \\
\hline
\end{tabular}


APPENDIX 3. Preoperative characteristics of patients excluded from matching

\begin{tabular}{|c|c|c|c|}
\hline Characteristic & $\begin{array}{c}\text { SU-AVR } \\
\mathrm{n}=\mathbf{7 8} \\
\end{array}$ & $\begin{array}{c}\text { TAVI } \\
\mathbf{n}=1671\end{array}$ & $P$ value \\
\hline Age (y) & $74.9 \pm 4.8$ & $82.2 \pm 5.8$ & $<.001$ \\
\hline Female gender & $50(64.1)$ & $100(59.8)$ & .45 \\
\hline Body mass index & $28.4 \pm 4.6$ & $25.6 \pm 4.4$ & $<.001$ \\
\hline Diabetes & $18(23.1)$ & $425(25.4)$ & .63 \\
\hline Arterial hypertension & $73(93.6)$ & $1377(82.4)$ & .01 \\
\hline Extracardiac arteriopathy* & $4(5.1)$ & $609(36.5)$ & $<.001$ \\
\hline Chronic lung disease* & $2(2.6)$ & $426(25.5)$ & $<.001$ \\
\hline Previous cardiac surgery* & $7(9.0)$ & $327(19.6)$ & .02 \\
\hline \multicolumn{4}{|l|}{ Renal impairment $(\mathrm{mL} / \mathrm{min} \text {; missing } \mathrm{n}=21)^{*}$} \\
\hline$>85$ & $61(78.2)$ & $55(3.3)$ & \\
\hline $51-84$ & $16(20.5)$ & $516(31.3)$ & $<.001$ \\
\hline$<51$ & $1(1.3)$ & $1021(61.9)$ & \\
\hline Dialysis & 0 & $58(3.5)$ & \\
\hline \multicolumn{4}{|l|}{ Pulmonary hypertension $(\mathrm{mm} \mathrm{Hg}) *$} \\
\hline No & $78(100)$ & $572(34.2)$ & $<.001$ \\
\hline $31-55$ & 0 & $895(53.6)$ & \\
\hline$>55$ & 0 & $204(12.2)$ & \\
\hline History of coronary artery disease as described below & 0 & $756(45.2)$ & $<.001$ \\
\hline Left ventricular ejection fraction $(\%)$ & $60.3 \pm 7.6$ & $53.0 \pm 12.4$ & $<.001$ \\
\hline Neurological dysfunction per Euroscore & 0 & $149(8.9)$ & .001 \\
\hline Logistic EuroSCORE & $6.8 \pm 4.2$ & $22.1 \pm 13.7$ & $<.001$ \\
\hline Aortic valve area $\left(\mathrm{cm}^{2}\right)$ & $0.54 \pm 0.23 / \mathrm{n}=11$ & $0.46 \pm 0.14 / \mathrm{n}=1390$ & .24 \\
\hline Peak aortic gradient $(\mathrm{mm} \mathrm{Hg})$ & $86.9 \pm 20.8$ & $81.4 \pm 22.5$ & .03 \\
\hline Mean aortic gradient $(\mathrm{mm} \mathrm{Hg})$ & $49.5 \pm 12.5$ & $50.1 \pm 14.9$ & .89 \\
\hline \multicolumn{4}{|l|}{ NYHA functional classification } \\
\hline I & $1(1.2)$ & $45(2.7)$ & \\
\hline II & $15(19.2)$ & 363 (15.7) & .17 \\
\hline III & $58(74.4)$ & $1150(68.8)$ & \\
\hline IV & $4(5.1)$ & $213(12.8)$ & \\
\hline
\end{tabular}

SU-AVR, Sutureless aortic valve replacement; TAVI, transcatheter aortic valve implantation; EuroSCORE, European system for cardiac operative risk evaluation; NYHA, New York Heart Association. *Per EuroSCORE II definition.

APPENDIX 4. Outcomes of patients excluded from matching

\begin{tabular}{lccc}
\hline \multicolumn{1}{c}{ Outcome } & $\begin{array}{c}\text { SU-AVR } \\
\mathbf{n}=\mathbf{7 8}\end{array}$ & $\begin{array}{c}\text { TAVI } \\
\mathbf{n}=\mathbf{1 6 7 1}\end{array}$ & $\boldsymbol{P}$ value \\
\hline Mortality & $1(1.3)$ & $126(7.5)$ & .04 \\
Lack of device success & $1(1.3)$ & $200(12.0)$ & .001 \\
Any stroke & $2(2.6)$ & $48(2.9)$ & $>.999$ \\
Bleeding (life-threatening or & $4(5.1)$ & $347(21.0)$ & $<.001$ \\
$\quad$ major) & & & \\
Pacemaker implantation & $6(7.7)$ & $110(6.6)$ & .70 \\
Any myocardial infarction & 0 & $26(1.6)$ & .62 \\
Severe paravalvular leakage & 0 & $87(5.2)$ & .03 \\
Paravalvular Leakage & & & \\
$\quad$ None & $78(100)$ & $1075(64.3)$ & $<.001$ \\
$\quad$ Mild & 0 & $509(30.5)$ & \\
$\quad$ Mild & 0 & $87(5.2)$ & \\
\hline
\end{tabular}

